\title{
Bienestar psicológico y discapacidad: una comparativa entre estudiantes universitarios
}

\author{
Francisco J. Alós Cívico ${ }^{1}$, Virginia I. Acedo ${ }^{1}$, \\ Miguel Ángel Maldonado Herves ${ }^{1}$, Eliana María Moreno \\ Osella $^{1}$
}

${ }^{1}$ Universidad de Córdoba

España

Correspondencia: Francisco J. Alós Cívico. Universidad de Córdoba. E-mail: ed1alcif@uco.es

(C) Universidad de Almería and Ilustre Colegio Oficial de la Psicología de Andalucía Oriental (Spain) 


\section{Resumen}

Introducción. La presencia de estudiantes con discapacidad en las universidades ha aumentado en los últimos años, sin embrago, aún son escasos los trabajos que estudian el bienestar psicológico en este colectivo. El objetivo de esta investigación es conocer el grado de bienestar psicológico en estudiantes universitarios con discapacidad y analizarlo de manera comparativa con una muestra de estudiantes sin discapacidad.

Método. Se aplica un diseño ex post facto prospectivo con grupo control en una muestra de 182 estudiantes, 72 con discapacidad y 110 sin discapacidad. Se administró la adaptación española de la escala de Bienestar Psicológico de Ryff.

Resultados. Ambos grupos presentan puntuaciones generales medias en la escala, sin embargo, se observan diferencias estadísticamente significativas entre los grupos tanto en la puntuación global en Bienestar Psicológico como en cuatro dimensiones de la escala: Relaciones Positivas, Autonomía, Dominio del Entorno y Crecimiento Personal.

Discusión y conclusiones. Este estudio aporta nuevos datos en un ámbito poco explorado. Se discuten aspectos clave para el desarrollo de estrategias de abordaje de dimensiones psicológicas en este colectivo y con un enfoque inclusivo en el contexto universitario.

Palabras Clave: Bienestar psicológico, universidad, discapacidad, estudiantes. 


\begin{abstract}
Introduction: The presence of students with disabilities in universities has increased in recent years, however, studies about psychological well-being of this group are still scarce. The objective of this research is to know and analyze the degree of psychological well-being in university students with disabilities and compare it with a sample of students without disabilities.

Method: A prospective ex post facto design with control group is applied in a sample of 182 students, 72 with disabilities and 110 without disabilities. The Spanish adaptation of the Ryff Psychological Welfare scale was administered.

Results: Both groups have average overall scores on the scale, however, statistically significant differences are observed between the groups. The overall score in Psychological Wellbeing is significantly lower in students with disabilities and in four dimensions of the scale: Positive Relations, Autonomy, Domain of the Environment and Personal Growth. In addition, differences were recorded regarding the sex variable in three dimensions.

Discussion or Conclusion: This study provides new data in a little explored area. Key aspects for the development of strategies for addressing psychological dimensions in this group and with an inclusive approach in the university context are discussed.
\end{abstract}

Keywords: psychological well-being, university, disability, students. 


\section{Introducción}

La Convención Internacional de los Derechos de las Personas con Discapacidad aprobada el 13 de diciembre de 2006 por Naciones Unidas, es el primer instrumento en el que se afirma, en su artículo 24, que todas las personas con cualquier tipo de discapacidad tienen derecho a la educación, sin discriminación y sobre la base de igualdad de oportunidades garantizando un sistema de educación inclusivo a todos los niveles, así como la enseñanza a lo largo de su vida. Por otra parte, a nivel comunitario, como marco de referencia, se recoge en la Carta de los Derechos Fundamentales de la Unión Europea el derecho a la educación con la prohibición de discriminación por razones de discapacidad (artículo 21). Discapacidad es un término general que abarca las deficiencias, las limitaciones de la actividad y las restricciones de la participación. Las deficiencias son problemas que afectan a una estructura o función corporal; las limitaciones de la actividad son dificultades para ejecutar acciones o tareas, y las restricciones de la participación son problemas para participar en situaciones vitales. Por consiguiente, este es un fenómeno complejo que refleja una interacción entre las características del organismo humano y las características de la sociedad en la que vive (Alcolea, 2017; OMS, 2011).

La marginación, exclusión y estigmatización son algunos de los problemas más graves que han enfrentado históricamente las personas con discapacidad, la preocupación por esta situación, y la firme voluntad de revertirla, está en el núcleo de la Convención sobre los Derechos de las Personas con Discapacidad. A partir de la creación de esta herramienta, existe una tendencia a reconocer que el principal obstáculo para el ejercicio de los derechos y la plena inclusión social no es la discapacidad en sí, sino las barreras en el entorno y en las actitudes con las que ésta interactúa. En el caso de las personas con discapacidad mental, estas barreras se constituyen por los límites que las diferentes culturas y sociedades imponen a los comportamientos humanos, así como por el estigma social y las consecuentes actitudes y prácticas discriminatorias de las que han sido y continúan siendo objeto (Fernández, 2017).

Desde un punto de vista académico y social, la presencia de estudiantes con discapacidad en las universidades se ha incrementado en los últimos años (Kendall, 2016). Según el III Estudio sobre inclusión de personas con discapacidad en el sistema universitario español (Fundación Universia, 2017), este alumnado representa un 1.7\% del total de los estudiantes universitarios en España. En la actualidad, las instituciones de educación superior 
están haciendo un esfuerzo por disminuir las barreras en el acceso y facilitar la inclusión de personas con discapacidad. Sin embargo, algunos estudios, como el de Soorenian (2013), encuentran que todavía muchos estudiantes con discapacidad sufren discriminación en la universidad, a pesar de que existen leyes y convenciones nacionales e internacionales que intentan regular estos aspectos. Por esta razón, en los últimos años diversos estudios se han interesado por la inclusión de este colectivo en la comunidad universitaria. Aunque las publicaciones son aún escasas, la literatura refleja algunos aspectos de la situación actual de este colectivo dentro de la universidad, como por ejemplo, las necesidades que presentan (Alcedo, Aguado, Real, González y Rueda, 2007); la búsqueda de la inclusividad (Alcantud, Ávila y Asensi, 2000; Barton, 2009; García-Cano, Alós, Jiménez y Polonio, 2018); el desarrollo de guías, recursos o programas orientados a su atención (González-Badía y Molina, 2006) o las actitudes hacia las personas con discapacidad (Díaz, 2002; Polo y López, 2005; Suriá, Bueno y Rosser, 2011).

La universidad es un contexto donde se dan situaciones potencialmente estresantes para los estudiantes, y normalmente representa un reto para los mismos (Carnicer y Calderón, 2014; Haynes, 2002; Kitzrow, 2003). Esta población está sometida constantemente a evaluaciones (académicas, personales y sociales), y por ello, su bienestar psicológico puede verse afectado (Velásquez et al., 2008). Muchos autores indican que estas dificultades son mayores para los estudiantes universitarios con discapacidad (Marshak,Van Wieren, Ferrell, Swiss, y Dugan, 2010; Titchkosky, 2011), porque no sólo se enfrentan a las exigencias propias de este contexto, sino que también tienen que superar barreras arquitectónicas (Fernández, Álvarez, y Malvar, 2012), curriculares (Álvarez-Pérez, Alegre de la Rosa y López-Aguilar, 2012), actitudinales (Suriá, 2014) y los propios obstáculos internos, que podrían afectar a su bienestar (Polo y López-Justicia, 2012).

El concepto de bienestar psicológico ha sido definido de dos formas diferentes: hedónica y eudaimónica. El hedonismo, concibe la relación de bienestar y felicidad, con presencia de afecto positivo y ausencia del negativo, siendo el concepto de bienestar subjetivo el predominante en esta perspectiva (Diener, 1984). Por otro lado, la perspectiva eudaimónica, con Aristóteles como uno de sus autores representativos, propone el bienestar como una forma de vivir plenamente y el desarrollo armónico del potencial humano (Deci y Ryan, 2006; Ryan, Huta y Deci, 2008). Según esta última perspectiva, una persona que experimenta un alto bienestar psicológico encuentra la vida satisfactoria y significativa, 
independientemente de la presencia o ausencia de altos niveles de emociones positivas y bajos niveles de emociones negativas (Tong, Lum, Sasaki y Yu, 2018). Una de las autoras más importantes en esta línea es Carol Ryff, que propone el término de bienestar psicológico entendiéndolo como el desarrollo del verdadero potencial de uno mismo. Ryff presenta un modelo explicativo multidimensional, y propone una escala (Psychological Well-Being Scales, PWBS) (Ryff, 1989a, 1995), que permite evaluar seis dimensiones del bienestar psicológico:

Autoaceptación es la capacidad de las personas de sentirse bien consigo mismas aun siendo conscientes de sus propias limitaciones, aceptando los aspectos positivos y negativos y valorando positivamente el pasado.

Relaciones positivas con otras personas se refieren a las relaciones con los demás como estables, estrechas y cálidas, tener amigos en los que pueda confiar, fuerte empatía y preocupación por el bienestar de los demás.

Autonomía es la capacidad para resistir a las presiones sociales, con la capacidad de mantener la individualidad ante diferentes contextos sociales, así como capacidad de autodeterminación, manteniendo su independencia y autoridad personal.

Dominio del entorno es la habilidad para crear o elegir contextos, sensación de control y de competencia, sabiendo sacar provecho de las oportunidades, capacidad para manejar eficazmente la vida y el mundo.

Propósito en la vida es la capacidad para establecer objetivos y marcarse metas en la vida, con la sensación de tener un rumbo y de que el presente y el pasado tienen sentido.

Crecimiento personal es el sentido de desarrollo continuo y crecimiento como persona, apertura a nuevas experiencias y sentido de realizar el potencial personal, verse a sí mismo progresando y con la capacidad para saber apreciar las mejoras personales.

Este modelo de bienestar psicológico ha sido ampliamente estudiado en Psicología, tanto en población general como en estudiantes universitarios (Amaya y Gaviaría, 2007; Chávez, 2006; Páramo, Straniero, García, Torrecilla y Gómez, 2012; Rosa-Rodríguez, 
Negrón, Maldonado, Toledo y Quiñones, 2015; Sandoval, Dorner y Véliz, 2017; Shah y Siddiqui, 2015; Soria, Martínez, Bresó, Llorens y Grau, 2005; Velásquez et al., 2008; Zubieta y Delfino, 2010). Sin embargo, son escasas las investigaciones que hayan tenido como objeto de estudio el ajuste psicológico y el grado de bienestar que presentan las personas con discapacidad o los estudiantes universitarios con esta condición (García-Cano et al., 2018; Suriá, 2017). Dentro de las investigaciones que abordan este último aspecto, encontramos estudios que comparan el grado de bienestar entre personas con o sin discapacidad. Samsari y Soulis (2019), encuentran que el bienestar subjetivo es significativamente menor en personas con discapacidad física en comparación con sujetos sin discapacidad. En esta misma línea, Pinquart y Pfeiffer (2011), reflejan una importante disminución del bienestar psicológico en personas con discapacidad visual frente a personas sin discapacidad. El estudio de Cheng y Sin (2020) compara esta variable entre estudiantes universitarios con y sin discapacidad auditiva, mencionan que la calidad de vida universitaria, junto con otras variables de bienestar psicológico son menores en los estudiantes con discapacidad auditiva. Con respecto al bienestar psicológico comparado según el género, Emerson et al. (2020) hallaron que las mujeres con discapacidad presentan mejores indicadores de bienestar que los hombres, principalmente en criterios como satisfacción con la vida, valía o felicidad.

La preocupación de las universidades por ofrecer una enseñanza de calidad supone considerar todas las variables implicadas en este proceso, entre las que se encuentra un buen ajuste psicológico. Conocer la percepción del bienestar psicológico de los estudiantes es importante para que se desarrollen futuras intervenciones y programas más acordes sus necesidades (Burris, Brechting, Salsman y Carlson, 2009; Cooke, Bewick, Barkham, Bradley y Audin, 2006; García-Cano et al. 2018). Tradicionalmente, los servicios específicos de atención a la diversidad han dedicado esfuerzos para disminuir barreras arquitectónicas y curriculares, $u$ ofrecer apoyo relacionado principalmente con aspectos académicos. Aunque se ha avanzado mucho en estos aspectos en los últimos años, es necesario a la vez, ampliar la cobertura de los servicios para abordar la atención de otro tipo de necesidades como, por ejemplo, promover el bienestar psicológico dentro de la universidad.

\section{Objetivos e hipótesis}

En un primer Diagnóstico de Inclusión de Personas con Discapacidad de la Universidad de Córdoba (García-Cano et al., 2018) se evaluaron distintos indicadores, entre los cuales se encontraban también algunas medidas de bienestar psicológico. Los resultados 
de este informe mostraron que en general, las mujeres con discapacidad presentaban mayor bienestar psicológico que los hombres, con un mayor ajuste en la dimensión de autoaceptación y propósito en la vida. A pesar de que las puntuaciones arrojadas por el alumnado con discapacidad se encontraban dentro de la media, se hace necesario profundizar acerca de si los niveles de bienestar serían similares al de estudiantes sin discapacidad. El objetivo de este estudio es comparar las puntuaciones obtenidas en la escala de Bienestar Psicológico en una muestra de estudiantes universitarios, con y sin discapacidad de la Universidad de Córdoba (España). Para ello y en base a los datos de estudios previos se platean las siguientes hipótesis de trabajo: Existirán menores indicadores de bienestar psicológico en estudiantes con discapacidad en comparación con aquellos sin discapacidad. Las diferencias encontradas en las distintas subescalas serán significativamente mas bajas en el grupo con discapacidad en la comparación entre grupos. Existirán diferencias significativas atendiendo a la variable género en las puntuaciones globales de bienestar y en las subescalas de Autoaceptación y propósito en la vida en el grupo de estudiantes con discapacidad.

\section{Método}

\section{Participantes}

La muestra está formada por 182 estudiantes de la Universidad de Córdoba- UCO (España) procedentes de distintas titulaciones (Ciencias Sociales y Jurídicas, Ciencias de la salud, Arte y humanidades, Ingeniería y arquitectura y Ciencias respectivamente). Entre ellos, 72 presentan un grado de discapacidad reconocido legalmente (igual o superior al 33\%) y 110 son estudiantes sin discapacidad.

La muestra de estudiantes con discapacidad representa el 51\% del total del colectivo de la Universidad, este grupo tiene una media de edad de 24.8 años (DT=6.28), el 56\% son mujeres y el $44 \%$ hombres. Atendiendo al tipo de discapacidad que presentan, el $49,5 \%$ presentan discapacidad física, el 16,5\% psíquica, el 13, 2\% sensorial visual, el 8,8\% sensorial auditiva, el 6,6\% orgánica y el 5,5\% restante presenta otro tipo de discapacidad. La media de edad del grupo control es de 23.3 años (DT=5.49), y el porcentaje de mujeres y hombres es, respectivamente, del $60 \%$ y $40 \%$.

\section{Instrumentos}

Para la recogida de datos se aplicaron los siguientes instrumentos de evaluación: 
Cuestionario socio-demográfico: este recogía datos sobre el sexo, la edad, el nivel académico, la titulación que cursan actualmente, el curso académico en el que están matriculados y el tipo y grado de discapacidad (si procedía).

Escala de Bienestar Psicológico de Ryff, en su versión breve de 29 ítems (Ryff, 1989a, 1989b; Van Dierendonck, 2004), y adaptada al español por Díaz et al. (2006). En total, la escala se compone de seis dimensiones de bienestar psicológico: autoaceptación (ítems 1, 7, 17 y 24), relaciones positivas (ítems $2,8,12,22$ y 25), autonomía (ítems 3, 4, 9, 13 y 18), dominio del entorno (ítems 5, 10, 14, 19 y 29), crecimiento personal (ítems 21, 26, 27 y 28), propósito en la vida $(6,11,15,16$ y 20). En ella se debe indicar en qué medida las afirmaciones presentadas son más adecuadas para describir su realidad, utilizando una escala tipo Likert del 1-6, donde 1 equivale a totalmente en desacuerdo, 2: bastante en desacuerdo, 3: algo en desacuerdo, 4: algo de acuerdo, 5: bastante de acuerdo y 6: totalmente de acuerdo. La escala presenta una buena consistencia interna con un valor $\alpha$ de Cronbach de 0,84 a 0,70 (Díaz et al., 2006).

\section{Procedimiento}

Se trata de un estudio ex post facto prospectivo con grupo control (León y Montero, 2015), donde los participantes dieron su consentimiento escrito para participar de la investigación.

Debido a la disponibilidad y accesibilidad de la muestra, la selección de los participantes se realizó de dos formas. En un primer momento se seleccionó al grupo de estudiantes con discapacidad a través de un muestreo de conveniencia entre el alumnado que presenta discapacidad en la Universidad de Córdoba. Posteriormente se les envió un correo con un enlace web para cumplimentar el consentimiento informado y el cuestionario de forma anónima. Para obtener el mayor número de participantes posibles, se realizaron llamadas telefónicas recordatorias para la cumplimentación del cuestionario. El grupo control fue seleccionado por muestreo accidental entre los y las estudiantes de las distintas titulaciones de la misma universidad. A quienes se les envió un correo con un enlace web para cumplimentar el consentimiento informado y el cuestionario de forma anónima. 


\section{Análisis de datos}

Tras la recogida de los datos, se analizó la homogeneidad de los grupos y se examinó la existencia de diferencias en las distintas dimensiones del cuestionario de Bienestar Psicológico. Se realizaron dos contrastes de medias con la prueba $t$ de Student y se calculó el estadístico de correlación eta con las dos variables de agrupación. Para el análisis de los datos se utilizó el programa estadístico SPSS versión 22. En relación a las variables utilizadas, se distinguen:

Variables de agrupación (VA): grupo de alumnado (VA1) (con discapacidad o sin discapacidad) y sexo (VA2) (hombre o mujer).

Variables dependientes (VD): puntuación en la dimensión Autoaceptación (VD1), puntuación en la dimensión Relaciones Positivas (VD2), puntuación en la dimensión Autonomía (VD3), puntuación en la dimensión Dominio del entorno (VD4), puntuación en la dimensión Crecimiento Personal (VD5), puntuación en la dimensión Propósito en la Vida (VD6) y puntuación total en el cuestionario Bienestar Psicológico (VD7).

\section{Resultados}

Se realizaron los análisis oportunos para comprobar si existían diferencias significativas entre los grupos de alumnos (discapacidad/ no discapacidad) en las variables de sexo $\left[\chi^{2}\right.$ $(1, \mathrm{~N}=182)=0.35, p=.55], \mathrm{y}$ edad $t(137.50)=1.691, p=.09$. En este estudio, el alfa de Cronbach de la escala utilizada tuvo un valor total de 0.85 .

A continuación, se presentan los resultados de los análisis descriptivos segregados por grupos, por ítems y por dimensiones de la escala Bienestar Psicológico (Tabla 1 y Tabla 2). 
Tabla 1. Comparación de Puntuaciones Obtenidas en los Ítems de la Escala de Bienestar Psicológico (Díaz et al., 2006) en Función de la Presencia o Ausencia de Discapacidad

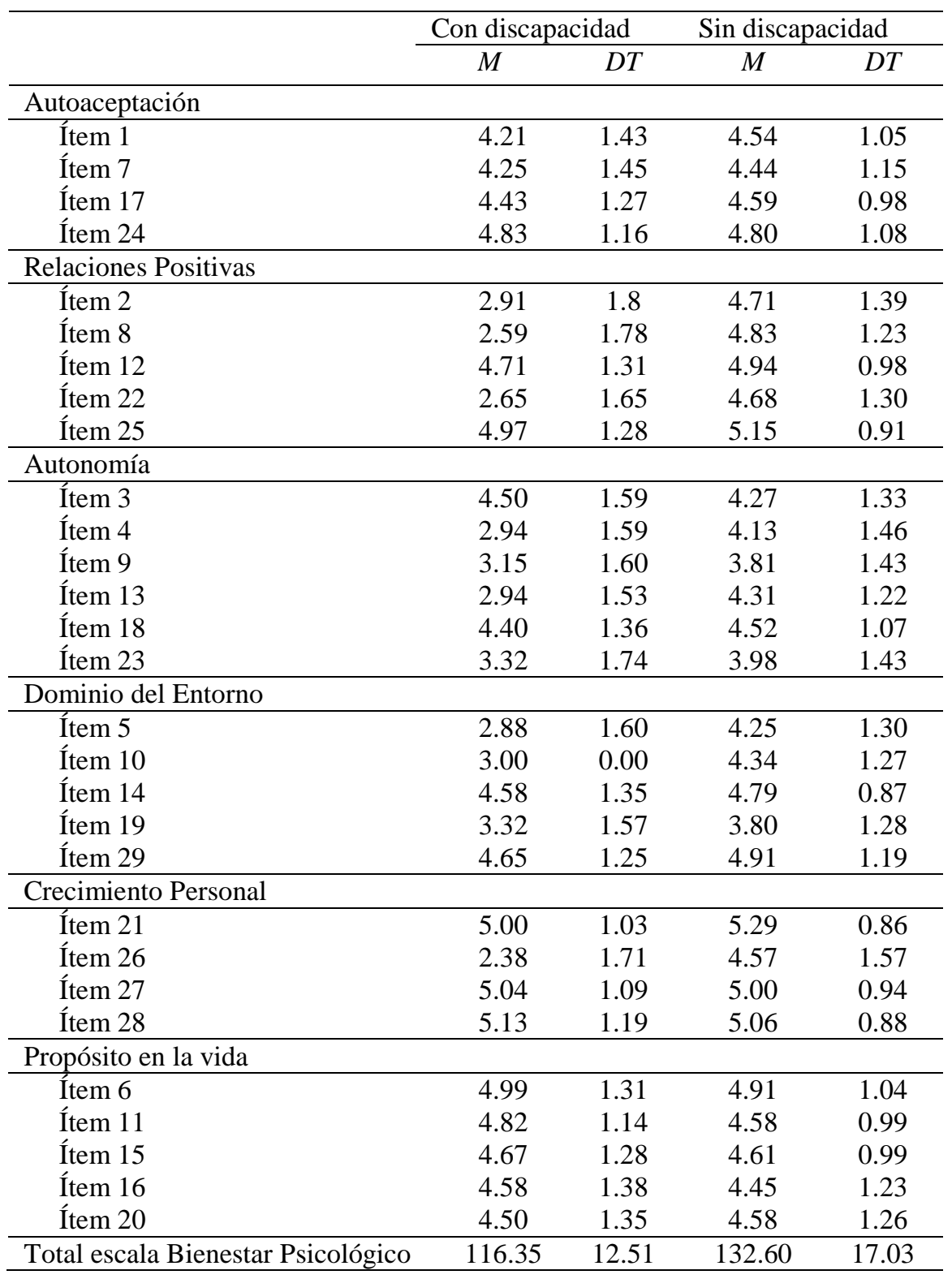

De forma general, las puntuaciones medias obtenidas por los participantes sin discapacidad son mayores en los ítems referentes a autoaceptación (a excepción del ítem 24), relaciones positivas, autonomía (a excepción del ítem 3), dominio del entorno y crecimiento personal (a excepción de los ítems, 27 y 28). Sin embargo, las puntuaciones medias obtenidas en los ítems relacionados con el propósito en la vida son mayores en los participantes con discapacidad (a excepción del ítem 20). Por tanto, la puntuación media total es mayor en las personas sin discapacidad. 
Tabla 2. Análisis Descriptivo de las Medias para cada Dimensión de la Escala Bienestar Psicológico Según la Presencia o Ausencia de Discapacidad y la Variable Sexo

\begin{tabular}{|c|c|c|c|c|c|c|c|c|c|c|c|c|}
\hline & \multicolumn{6}{|c|}{ Con discapacidad } & \multicolumn{6}{|c|}{ Sin discapacidad } \\
\hline & \multicolumn{2}{|c|}{ Hombres } & \multicolumn{2}{|c|}{ Mujeres } & \multicolumn{2}{|c|}{ Total } & \multicolumn{2}{|c|}{ Hombres } & \multicolumn{2}{|c|}{ Mujeres } & \multicolumn{2}{|c|}{ Total } \\
\hline & $M$ & $S D$ & $M$ & $S D$ & $M$ & $S D$ & $M$ & $S D$ & $M$ & $S D$ & $M$ & $S D$ \\
\hline $\begin{array}{l}\text { Autoacepta- } \\
\text { ción }\end{array}$ & 16.03 & 4.11 & 19.08 & 6.65 & 17.72 & 4.13 & 17.91 & 3.71 & 18.51 & 3.42 & 18.27 & 3.53 \\
\hline $\begin{array}{l}\text { Relaciones } \\
\text { Positivas }\end{array}$ & 18.50 & 4.13 & 17.30 & 3.01 & 17.83 & 3.57 & 23.57 & 4.35 & 24.67 & 4.18 & 24.22 & 4.26 \\
\hline Autonomía & 21.91 & 5.04 & 20.75 & 3.57 & 21.26 & 4.30 & 26.00 & 3.72 & 24.37 & 5.07 & 25.03 & 4.63 \\
\hline $\begin{array}{l}\text { Dominio del } \\
\text { Entorno }\end{array}$ & 19.09 & 3.33 & 17.90 & 2.56 & 18.43 & 2.97 & 21.41 & 3.52 & 22.48 & 3.36 & 22.05 & 3.45 \\
\hline $\begin{array}{l}\text { Crecimiento } \\
\text { Personal }\end{array}$ & 17.50 & 3.21 & 17.58 & 2.37 & 17.54 & 2.76 & 18.91 & 3.11 & 20.53 & 2.72 & 19.88 & 2.98 \\
\hline $\begin{array}{l}\text { Propósito en la } \\
\text { vida }\end{array}$ & 22.38 & 6.04 & 24.50 & 4.86 & 23.56 & 5.48 & 22.07 & 4.50 & 23.85 & 3.54 & 23.14 & 4.03 \\
\hline Total escala & 115.4 & 13.8 & 117.1 & 11.4 & 116.3 & 12.5 & 129.8 & 16.5 & 134.4 & 17.2 & 132.6 & 17.0 \\
\hline $\begin{array}{l}\text { Bienestar } \\
\text { Psicológico }\end{array}$ & 1 & 4 & 0 & 7 & 4 & 1 & 6 & 4 & 2 & 4 & 0 & 3 \\
\hline
\end{tabular}

Si analizamos estos datos por sexo, los participantes con discapacidad (mujeres) obtienen puntuaciones medias mayores en las dimensiones de autoaceptación, crecimiento personal y propósito en la vida, frente a mayores puntuaciones medias de participantes con discapacidad (hombres) en las dimensiones de relaciones positivas, autonomía y dominio del entorno. Sin embargo, realizando esta misma comparación por sexo en personas sin discapacidad, las mujeres obtienen mayores puntuaciones medias en las dimensiones de autoaceptación, relaciones positivas, dominio del entorno, crecimiento personal y propósito en la vida, frente a la única dimensión en la que los hombres obtienen mayores puntuaciones medias, la autonomía. Por lo tanto, en ambos casos (con discapacidad o sin discapacidad) las mujeres obtienen una mayor puntuación media total teniendo en cuenta todas las dimensiones medidas en comparación con los hombres.

Los datos comentados pueden observarse en la figura 1 donde se reflejan las medias de las puntuaciones totales en el cuestionario Bienestar Psicológico, teniendo en cuenta las dos variables de agrupación contempladas en este estudio. 


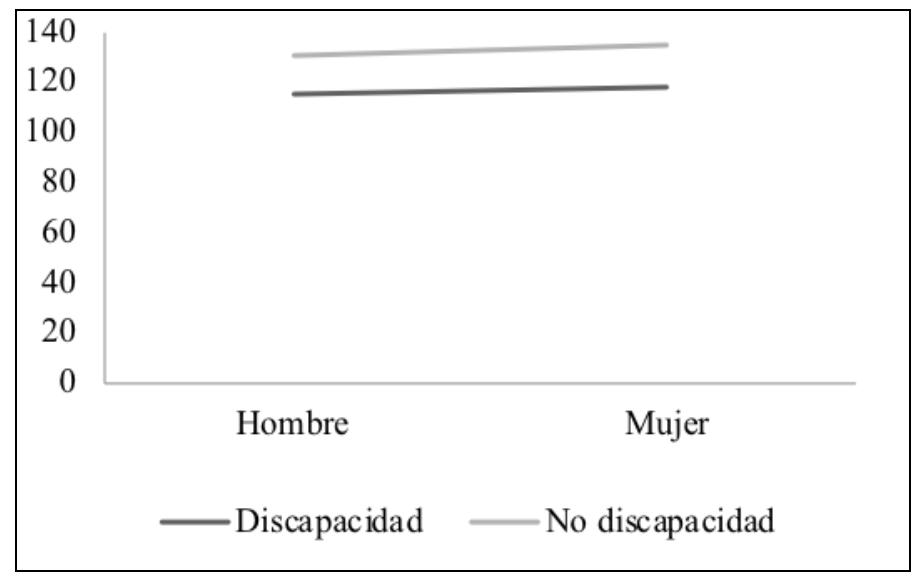

Figura 1. Puntuación total del cuestionario Bienestar Psicológico en función del sexo y el grupo de estudiantes (discapacidad o no discapacidad)

Para conocer si existían diferencias entre los grupos (con y sin discapacidad) en las puntuaciones totales y en cada una de las dimensiones, se realizó en primer lugar una prueba de normalidad (Kolmogorov-Smirnov) del total de puntuaciones medias de las dimensiones de la escala de bienestar psicológico, cuyos datos fueron significativos $(p<.001)$ (ver Tabla 3). Para poder analizar y comparar los datos se realizó una prueba no paramétrica mediante una comparación de medias con una U de Mann-Whitney para muestras independientes. Los datos reflejan que existen diferencias significativas $(p<.001)$ en la puntuación total de la escala Bienestar Psicológico $(p=.000)$ y en las dimensiones de Relaciones Positivas $(\mathrm{p}=.000)$, Autonomía $(p=.000)$, Dominio del Entorno $(p=.000)$ y Crecimiento Personal $(p=.000)$ (Ver Tabla 4). Por lo tanto, tras los datos hallados mediante los análisis estadísticos, se observa una diferencia estadísticamente significativa, con mayores puntuaciones en la escala de bienestar psicológico de las personas sin discapacidad frente a las personas con discapacidad.

Para conocer si existían diferencias en función del sexo en las puntuaciones obtenidas en las distintas variables, se realizó una prueba no paramétrica de comparación de medias con la prueba U de Mann-Whitney para muestras independientes. Los datos muestran que existen diferencias significativas en las puntuaciones de las dimensiones de Autoaceptación ( $p=$ $.005)$, Crecimiento Personal ( $p=.041)$ y Propósito en la Vida $(p=.019)$ y atendiendo dichas diferencias a mayores puntuaciones en mujeres que en hombres (Ver Tabla 4). 
Tabla 3. Prueba de normalidad de Kolmogorov-Smirnov

\begin{tabular}{|c|c|c|c|c|}
\hline \multicolumn{5}{|c|}{ Pruebas de normalidad } \\
\hline & \multirow{2}{*}{ Discapacidad } & \multicolumn{3}{|c|}{ Kolmogorov-Smirnov ${ }^{\mathrm{a}}$} \\
\hline & & Estadístico & $\mathrm{gl}$ & Sig. \\
\hline \multirow{2}{*}{ Autoaceptación } & Con Discapacidad & ,126 & 72 &, 006 \\
\hline & Sin Discapacidad &, 142 & 110 &, 000 \\
\hline \multirow{2}{*}{ Relaciones Positivas } & Con Discapacidad &, 182 & 72 &, 000 \\
\hline & Sin Discapacidad &, 108 & 110 &, 003 \\
\hline \multirow{2}{*}{ Autonomía } & Con Discapacidad &, 122 & 72 &, 010 \\
\hline & Sin discapacidad &, 068 & 110 &, $200^{*}$ \\
\hline \multirow{2}{*}{ Dominio del Entorno } & Con Discapacidad & ,085 & 72 &, $200^{*}$ \\
\hline & Sin Discapacidad &, 111 & 110 &, 002 \\
\hline \multirow{2}{*}{ Crecimiento Personal } & Con Discapacidad &, 149 & 72 &, 000 \\
\hline & Sin Discapacidad &, 100 & 110 & ,009 \\
\hline \multirow{2}{*}{ Propósito en la Vida } & Con Discapacidad &, 185 & 72 &, 000 \\
\hline & Sin Discapacidad &, 116 & 110 &, 001 \\
\hline Total Escala Bienestar & Con Discapacidad &, 047 & 72 & $200^{*}$ \\
\hline Psicológico & Sin Discapacidad &, 078 & 110 &, 102 \\
\hline
\end{tabular}

*. Esto es un límite inferior de la significación verdadera.

a. Corrección de significación de Lilliefors

Tabla 4. Comparación de medias mediante prueba no paramétrica (U de Mann-Whitney para muestras independientes) para cada dimensión de la Escala Bienestar Psicológico según las variables discapacidad (Presencia o Ausencia de Discapacidad) y sexo (hombre o mujer)

\begin{tabular}{lcc}
\hline & \multicolumn{2}{c}{ Prueba U de Mann-Whitney } \\
\hline Dimensiones & Discapacidad & Género \\
\hline Autoaceptación & Sig. & Sig. \\
Relaciones Positivas & .480 & $.005^{* *}$ \\
Autonomía & $.000^{* *}$ & .556 \\
Dominio del Entorno & $.000^{* *}$ & .055 \\
Crecimiento Personal & $.000^{* *}$ & .838 \\
Propósito en la vida & $.000^{* *}$ & $.041^{*}$ \\
\hline Total escala Bienestar Psicológico & .078 & $.019^{*}$ \\
\hline
\end{tabular}

$* \mathrm{p}<.05, * * p<.01$ 


\section{Discusión y Conclusiones}

Este estudio refleja que existen diferencias estadísticamente significativas en función de la presencia o ausencia de discapacidad en la puntuación total de la escala de Bienestar Psicológico. En líneas generales, los datos confirman la primera hipótesis de trabajo y muestran que el alumnado con discapacidad presenta puntuaciones más bajas en la escala global que aquellos sin discapacidad. Esto coincide con los datos expuestos en otros estudios que comparan niveles de bienestar en población general con o sin discapacidad física (Nosek, Hughes, Swedlund, Taylor y Swank, 2003; Samsari y Soulis, 2019) o visual (Pinquart y Pfeiffer, 2011), y con los resultados de Cheng y Sin (2020) en estudiantes universitarios con y sin discapacidad auditiva.

Es importante considerar que, en los resultados descriptivos de la escala, ambos grupos arrojan puntuaciones medias, es decir, de forma global, el alumnado universitario con y sin discapacidad presenta un nivel medio de bienestar psicológico, estos resultados están en la línea de otros estudios realizados (García-Cano et al., 2018). La escala breve de Bienestar Psicológico adaptada al español es un instrumento ampliamente utilizado y consolidado (Díaz et al., 2006). Sin embrago, no existen baremos ni puntos de corte establecidos para población española que permitan discriminar con exactitud si la puntuación obtenida indica un buen o mal ajuste del bienestar psicológico. Además, son escasas las investigaciones que recojan puntuaciones de bienestar psicológico en población de estudiantes con discapacidad y aún menores aquellas que comparen las puntuaciones con otro grupo de referencia (García-Cano et al., 2018; Suriá, 2017). Esto genera limitaciones para llegar a conclusiones sólidas en este campo. Los datos de esta investigación nos permiten conocer los niveles de bienestar psicológico de las personas con discapacidad que cursan estudios universitarios, en comparación con un grupo de pares de referencia, lo cuál supone un aporte esencial del presente estudio.

Atendiendo a las distintas dimensiones de la escala y siguiendo en la comparación entre grupos, se comprueba de manera parcial la segunda hipótesis de trabajo, observando puntuaciones significativamente mas bajas en estudiantes con discapacidad sólo en cuatro de las seis dimensiones: Relaciones Positivas, Autonomía, Dominio el Entorno y Crecimiento Personal. Dentro de estas, los valores más bajos se encuentran en la dimensión asociada a Relaciones Positivas con otras personas, donde destacan los ítems que reflejan sentimientos de soledad, pocas personas con las cuales hablar y carencia de relaciones cercanas y de confian- 
za. Esto sigue la tendencia de los hallazgos de Dong y Lucas (2014) o Ekelman, Bazyk y Bazyk (2013), que reflejan que el apoyo social y el deseo de pertenecer a un grupo o actividad compartida es un elemento mediador en el bienestar subjetivo de estudiantes con distintos tipos de discapacidad. Junto con esto, una revisión sistemática de 58 estudios encuentra también una asociación consistente entre el apoyo social percibido y el bienestar en personas con discapacidad física (Tough, Siegrist y Fekete, 2017). Otros autores subrayan el papel que desempeña el apoyo social percibido en la mejora del bienestar, e inciden, en el hecho de que el medio social más próximo es el lugar donde deberían encontrarse esos apoyos (Martínez, 2016). En este sentido, la universidad representa un elemento importante del entorno social y natural de los y las estudiantes con discapacidad, gran parte de la vida cotidiana, de las interacciones sociales, del desarrollo académico profesional y de la orientación hacia un proyecto de vida se construyen en este contexto social próximo. Por lo que cobra sentido que dentro de la universidad se articulen estrategias orientadas a cubrir estas necesidades interpersonales y psicológicas.

Otra dimensión significativamente inferior entre estudiantes con discapacidad fue la de autonomía (que se asocia con la aptitud para resistir a las presiones sociales, la capacidad de autodeterminación y la autoridad personal), donde destacan los ítems asociados a la preocupación por la evaluación e influencia externa. El Dominio del Entorno es la capacidad para elegir o crear entornos favorables con el fin de satisfacer metas y necesidades, aquí los ítems que presentan puntuaciones más bajas indicarían dificultades para dirigir la propia vida hacia cambios y modos de vida satisfactorios, junto con dificultades para afrontar las demandas de la vida cotidiana. Por último, la dimensión de Crecimiento Personal, que se asocia a la realización del potencial personal, reflejaría principalmente la percepción de no haber mejorado mucho como persona en los últimos años y no conseguir el desarrollo pleno de su potencial.

El conocimiento de las dimensiones que reflejan valores más bajos permitiría diseñar entornos de aprendizaje y estrategias activas dirigidas a mejorar estos aspectos. Por lo que, se deberían tener en cuenta los datos hallados en este estudio, para desarrollar dichas estrategias de manera más eficaz, ya que estas podrían tener un efecto favorecedor sobre las distintas dimensiones del bienestar psicológico de las personas con discapacidad, aumentando así un mayor y mejor ajuste psicosocial en relación con su entorno. Estas estrategias podrían ir enfocadas a facilitar interacciones más positivas y de confianza entre estudiantes u otras personas significativas del entorno, tanto dentro como fuera del aula; el aumento de la participación de 
las personas con discapacidad en la vida universitaria que faciliten la expresión de opiniones y puntos de vista personales (Moriña, 2015); brindar apoyos específicos dentro de los servicios de atención universitarios, ofrecer orientación y apoyo para la consecución de metas personales significativas o incorporar intervenciones psicoeducativas dirigidas a promover el desarrollo personal. Estrategias de este tipo, podrían contribuir a atender las necesidades psicológicas detectadas en este colectivo, además de mejorar el desarrollo de todo su potencial y aumentar el bienestar general dentro del mundo universitario (Vázquez et al., 2008).

Por otro lado, que las instituciones académicas, así como el colectivo de estudiantes conozcan estos datos de manera concreta, podría propiciar una mayor concienciación sobre el peor bienestar psicológico que las personas con discapacidad sufren y por lo tanto de las barreras psicosociales existentes, pudiendo favorecer una mayor red de apoyo social que disminuya parte de sus efectos negativos.

Los análisis realizados atendiendo a la variable sexo, indican que no existen diferencias estadísticamente significativas entre hombres y mujeres en la puntuación total del cuestionario Bienestar Psicológico. En la línea de los hallazgos de Hamdan y Marmash (2007) y Shah y Siddiqui (2015). Esto nos llevaría a descartar gran parte de la tercera hipótesis de trabajo. La misma se apoyaba en los hallazgos de un estudio previo en el mismo contexto universitario, donde se encontraron diferencias en el bienestar entre hombres y mujeres con discapacidad y más específicamente, mejores puntuaciones de las mujeres en las dimensiones de autoaceptación y propósito en la vida (García-Cano et al., 2018). En este sentido se ha podido comprobar una tendencia en los datos, pero la misma no es estadísticamente significativa. Teniendo en cuenta las subdimensiones de la escala, en este estudio se encuentran diferencias en tres de ellas, Autoaceptación, Crecimiento Personal y Propósito en la Vida, siendo las mujeres las que obtienen mejores puntuaciones. En el grupo de mujeres con discapacidad únicamente se encuentran puntuaciones significativamente mayores en la dimensión de Crecimiento Personal, en comparación con los hombres de su mismo grupo, con un índice de asociación moderado. Algunos estudios recientes apoyan la idea de que existiría una interacción significativa entre discapacidad y género (Arciuli, Emerson y Llewellyn, 2019; Emerson et al., 2020). Sin embargo, los análisis en este sentido son escasos y no del todo concluyentes, por lo que se hace necesario profundizar en el estudio de esta variable e incorporar una perspectiva de género en el diseño de investigaciones posteriores. 
En líneas generales, el hecho de comprobar que los estudiantes con discapacidad presentan niveles de bienestar significativamente más bajos que sus pares, nos invita a reflexionar sobre las necesidades de este grupo y el papel que podrían desempeñar las políticas y, más específicamente, los servicios de atención a la diversidad en el abordaje de este tema.

\section{Conclusiones}

Las universidades españolas están implementando programas y medidas para la población con discapacidad. El impulso de los servicios específicos de apoyo en las universidades para distintos colectivos ha contribuido a que se lleven a cabo importantes medidas como, por ejemplo, la eliminación de barreras arquitectónicas (Polo y López, 2005), la creación de servicios de atención inclusiva y el reforzamiento de planes de inclusión, entre otras (Fundación Universia, 2017). Sin embargo, las dimensiones psicológicas o los problemas de corte relacional, como el bienestar psicológico, aún son escasamente atendidas (Alcedo et al., 2007, Burris et al., 2009; Cooke, Bewick, Barkham, Bradley y Audin, 2006; García-Cano et al., 2018). Las diferencias acerca del bienestar psicológico identificadas en este estudio ponen de manifiesto la importancia de ampliar (o consolidar) la cobertura de los servicios de apoyo universitarios para atender a estas necesidades. A nivel aplicado, los datos recogidos en este estudio podrían ayudar a que instituciones como las universitarias, adopten un enfoque preventivo y de promoción del bienestar psicosocial, lo cual tendría implicaciones directas sobre una mayor igualdad y la plena inclusión de las personas con discapacidad en el ámbito universitario.

\section{Limitaciones}

Una de las principales limitaciones de este estudio se refiere a la muestra, que fue recogida en una sola universidad, por ello, la generalización de los resultados debe tomarse con cautela. En futuras investigaciones sería adecuado ampliar la muestra y la localización geográfica de la misma, realizando, por ejemplo, un muestreo aleatorio estratificado, para poder comprobar si esta misma tendencia se da en otros contextos. A pesar de estas limitaciones, este trabajo contribuye a un campo poco explorado, y aporta datos que podrían ayudar a desarrollar el potencial de estudiantes universitarios con discapacidad a través de la implementación de estrategias de manera más eficaz, que favorezcan un aumento del bienestar psicológico en todas sus dimensiones. 


\section{Referencias}

Alcantud, F., Ávila, V. y Asensi, C. (2000). La Integración de Estudiantes con Discapacidad en los estudios superiores. Valencia: Universitat de València Estudi General (Servei de Publicacions).

Alcedo, M., Aguado, A., Real, S., González, M. y Rueda, B. (2007). Una revisión actualizada de la situación de los estudiantes con discapacidad en la universidad. Anuario de Psicología Clínica y de la Salud, 3, 7-18.

Alcolea Fernández, J. (2017). Cuidadores familiares de personas con discapacidad intelectual. Acción Social. Revista de política social y servicios sociales 1 (6), 108-117.

Álvarez-Pérez, P. R., Alegre-de-la-Rosa, O. M. y López-Aguilar, D. (2012). Las dificultades de adaptación a la enseñanza universitaria de los estudiantes con discapacidad: un análisis desde un enfoque de orientación inclusiva. Relieve. Revista Electrónica de Investigación y Evaluación Educativa, 18(2), 1-18. doi:10.7203/relieve.18.2.1986

Amaya, M. E. y Gaviaría, A. M. (2007). Estilos de vida, bienestar psicológico y sentido de coherencia en los estudiantes adultos de la universidad de San Buenaventura, seccional Medellín. Revista USB Medellín, 7(2), 199-385.

Arciuli, J., Emerson, E., y Llewellyn, G. (2019). Adolescents' self-report of school satisfaction: The interaction between disability and gender. School Psychology, 34(2), 148.doi:org/10.1037/spq0000275.

Barton, L. (2009). Estudios sobre discapacidad y la búsqueda de la inclusividad. Revista de Educacion, 349, 137-152.

Burris, J. L., Brechting, E. H., Salsman, J. y Carlson, C. R. (2009). Factors associated with the psychological well-being and distress of university students. Journal of American College Health, 57(5), 536-544. doi: 10.3200/JACH.57.5.536-544

Carnicer, J. G. y Calderón, C. (2014). Empatía y estrategias de afrontamiento como predictores del bienestar en estudiantes universitarios españoles. Electronic Journal of Research in Educational Psychology, 12(1), 129-146. doi:10.14204/ejrep.32.13117

Chávez, A. (2006). Bienestar Psicológico y su Influencia en el Rendimiento Académico de Estudiantes de Nivel Medio Superior. (Tesis de maestría). Recuperada de http://digeset.ucol.mx/tesis_posgrado/Pdf/ALFONSO_CHAVEZ_URIBE.pdf

Cheng, S., y Sin, K. F. (2020). University Self-efficacy and Quality of University Life among Students with and without Hearing Impairment and Hearing Students. International Journal of Disability, Development and Education, 1-13. doi:org/10.1080/1034912X.2020.1801989. 
Cooke, R., Bewick, B. M., Barkham, M., Bradley, M. y Audin, K. (2006) Measuring, monitoring and managing the psychological well-being of first year university students. British Journal of Guidance y Counselling, 34(4), 505-517. doi: 10.1080/03069880600942624

Deci, E. L. y Ryan, R. M. (2006). Edonia, Eudaimonia, and Well-being: an Introduction. Journal of Happiness Studies, 9, 139-170. doi:10.1007/s10902-006-9018-1

Diener, E. (1984). Subjetive Well-Being. Psichological Bulletin. 95(3), 542-575. doi:10.1037/00332909.95.3.542

Díaz, E. (2002). El factor actitudinal en la atención a la diversidad. Profesorado, revista de currículum y formación del profesorado, 6 (1-2), 151-165.

Díaz, D., Rodríguez-Carvajal, R., Blanco, A., Moreno-Jiménez, B., Gallardo, I., Valle, C. y Van Dierendonck, D. (2006). Adaptación española de las escalas de bienestar psicológico de Ryff. Psicothema, 18(3), 572-577.

Dong, S., y Lucas, M. S. (2014). Psychological profile of university students with different types of disabilities. Journal of College Student Development. 55(5), 481-485.doi: doi.org/10.1353/csd.2014.0044

Ekelman, B., Bazyk, S., y Bazyk, J. (2013). The relationship between occupational engagement and well-being from the perspective of university students with disabilities. Journal of Occupational Science, 20(3), 236 252. doi.org/10.1080/14427591.2012.716360.

Emerson, E., Fortune, N., Aitken, Z., Hatton, C., Stancliffe, R., y Llewellyn, G. (2020). The wellbeing of working-age adults with and without disability in the UK: Associations with age, gender, ethnicity, partnership status, educational attainment and employment status. Disability and Health Journal, 100889. https://doi.org/10.1016/j.dhjo.2020.100889.

Fernández, M. T. (2017). La discapacidad mental o psicosocial y la Convención sobre los Derechos de las Personas con Discapacidad. Revista de derechos humanos, Dfensor 11, 10-17.

Fernández, M. D., Álvarez, Q. y Malvar, M. L. (2012). Accesibilidad e inclusión en el Espacio Europeo de Educación Superior: el caso de la Universidad de Santiago de Compostela. Aula Abierta, 40(3), 71-82.

Fundación Universia (2017). Universidad y discapacidad. III Estudio sobre el grado de inclusión del sistema universitario español respecto de la realidad de la discapacidad. Recuperado de http://riberdis.cedd.net/bitstream/handle/11181/5199/Universidad_y_discapacidad_III_Estudio .pdf?sequence $=1 \mathrm{yrd}=0031541654123808$

García-Cano, M., Castillejo, A., Jiménez-Luque, N., Martínez-Gallego, I., Chiara, M. y Alós, F. (2017). Universidad y Discapacidad: Diagnóstico sobre la inclusión de estudiantes con disca- 
pacidad en la Universidad de Córdoba. Docencia y Derecho, Revista para la docencia jurídica universitaria, 11, 1-17.

García-Cano, M., Alós, F. J., Jiménez-Luque, N. y Polonio, G. (2018). I Diagnóstico sobre la inclusión de las personas con discapacidad en la Universidad de Córdoba. Córdoba: UCOPRESS, Editorial Universidad de Córdoba.

González, R., Montoya, I., Casullo, M. M., Verdú, B. y Bernabéu, J. (2002). Relación entre estilos y estrategias de afrontamiento y bienestar psicológico en adolescentes. Psicothema, 14(2), 363368.

González-Badía, J. y Molina, C. (2006). Universidad y Discapacidad. Guía de recursos. Colección Telefónica Accesible. Madrid: Cinca, SA.

Hamdan-Mansour, A. y Marmash, L. (2007). Psychological well-being and general health of jordanian university students. Journal of Psychosocial Nursing y Mental Health Services, 45(10), 31-39.

Haynes, N.M. (2002). Addressing students' social and emotional needs: The role of mental health teams in schools. Journal of Health $y$ Social Policy, 16(1-2), 109-123. doi:10.1300/J045v16n01_10

Kendall, L. (2016). Higher education and disability: Exploring student experiences. Cogent Education, 3(1). doi:10.1080/2331186X.2016.1256142

Kitzrow, M. (2003). The mental health needs of today's college students: Challenges and recommendations. National Association of Student Personnel Administrators, 41, 167-181.

León, O. G. y Montero, I. (2015). Métodos de Investigación en Psicología y Educación. Las Tradiciones Cuantitativa y Cualitativa (4a ed.). Madrid: McGraw-Hill.

Martínez, R. S. (2016). Bienestar subjetivo, resiliencia y discapacidad. Acciones e investigaciones sociales, (36), 113-140.

Marshak, L., Van Wieren, T., Ferrell, D. R., Swiss, L. y Dugan, C. (2010). Exploring barriers to college student use of disability services and accommodations. Journal of Postsecondary Education and Disability, 22, 151-165.

Moriña, A. (2015). Inclusive university settings? An analysis from the perspective of students with disabilities/¿ Contextos universitarios inclusivos? Un análisis desde la voz del alumnado con discapacidad. Cultura y Educación, 27(3), 669-694.

Nosek, M. A., Hughes, R. B., Swedlund, N., Taylor, H. B., y Swank, P. (2003). Self-esteem and women with disabilities. Social science y medicine, 56(8), 1737-1747.doi.org/10.1016/S02779536(02)00169-7 
OMS Organización Mundial de la Salud (2011). Informe mundial sobre la discapacidad. Suiza: Banco Mundial.

Páramo, M. A., Straniero, C. M., García, C. S., Torrecilla, N. M. y Gómez, E. E. (2012). Bienestar psicológico, estilos de personalidad y objetivos de vida en estudiantes universitarios. Pensamiento Psicológico, 10(1), 7-21.

Pinquart, M., y Pfeiffer, J. P. (2011). Psychological well-being in visually impaired and unimpaired individuals: a meta-analysis. British Journal of Visual Impairment, 29(1), 2745.doi.org/10.1177\%2F0264619610389572.

Polo, M. T. y López, M. D. (2005). Barreras de acceso al medio físico de los estudiantes con discapacidad motora de la Universidad de Granada. Electronic Journal of Research in Educational Psychology, 3(3), 121-132.

Polo, M. T. y López, M. D. (2011). Actitudes hacia las personas con discapacidad de estudiantes de la Universidad de Granada. Revista Española de Orientación y Psicopedagogía, 17(2), 195-211. doi: 10.5944/reop.vol.17.num.2.2006.11346

Polo, M. T. y López-Justicia, M. D. (2012). Autoconcepto de estudiantes universitarios con discapacidad visual, auditiva y motora. Revista Latinoamericana de Psicología, 44(2), 87-98.

Rodríguez Álvarez, V. (2013). Fuentes de información sobre discapacidad y empleo en España. Revista Española de Discapacidad, 1 (1): 73-95. doi: http://dx.doi.org/10.5569/2340-5104.01.01.04

Rosa-Rodríguez, Y., Negrón, C. N., Maldonado P. Y., Toledo O. N. y Quiñones, B. A. (2015). Dimensiones de bienestar psicológico y apoyo social percibido con relación al sexo y nivel de estudio en universitarios. Avances en Psicología Latinoamericana, 33(1), 31-43. doi:10.12804/ap133.01.2015.03

Ryan, R. M., Huta, V. y Deci, E. L. (2008). Living well: A self-determination theory perspective on eudaimonia. Journal of Happiness Studies, 9, 139-170. doi:10.1007/s10902-006-9023-4

Ryff, C. (1989a). Beyond Ponce de Leon and life satisfaction: New directions in quest of successful aging. International Journal of Behavioral Development, 12, 35-55.

Ryff, C. (1989b). Happiness is everything, or is it? Explorations on the meaning of psychological well-being. Journal of Personality and Social Psychology, 57, 1069-1081.

Ryff, C. D. (1995). Psychological Well-Being in Adult Life. Current Directions in Psychological Science, 4(4), 99-104.

Samsari, E. P., y Soulis, S. G. (2019). Problem Solving and Resilience Self-Efficacy as Factors of Subjective Well-Being in Greek Individuals with and without Physical Disabilities. International Journal of Special Education, 33(4), 938-953. 
Sandoval, S., Dorner, A. y Véliz, A. (2017). Bienestar psicológico en estudiantes de carreras de la salud. Investigación en Educación Médica, 6(24), 260-266. doi:10.1016/j.riem.2017.01.004

Shah, I. y Siddiqui, F. A. (2015). Gender Difference of Psychological Well Being among University Students. Journal of Asian Development Studies, 4(3), 110-116.

Soorenian, A. (2013). Disabled international students in British higher education. Rotterdam: Sense Publishers.

Soria, M. S., Martínez, I. M., Bresó, E., Llorens, S. y Grau, R. (2005). Bienestar psicológico en estudiantes universitarios: facilitadores y obstaculizadores del desempeño académico. Anales de Psicología, 21(1), 170-180.

Suriá, R. (2014). Actitudes integradoras en el contexto educativo de los estudiantes con discapacidad según la percepción de sus progenitores. Bordón. Revista de Pedagogía, 66, 157-172. doi:10.13042/Bordon.2014.66410

Suriá, R. (2017). Inteligencia emocional y bienestar subjetivo en estudiantes universitarios con diferentes tipos y grados de discapacidad. Bordón. Revista de Pedagogía, 70(1), 125-140. doi:10.13042/Bordon.2018.48584

Suriá, R., Bueno, A. y Rosser, A. (2011). Prejuicios entre los estudiantes hacia las personas con discapacidad: reflexiones a partir del caso de la Universidad de Alicante. Alternativas. Cuadernos de Trabajo Social, 18, 75-90. doi:10.14198/ALTERN2011.18.04

Titchkosky T. (2011). The question of access: disability, space, meaning. Toronto: University of Toronto Press.

Tong, E. M. W., Lum, D. J. K., Sasaki, E. y Yu, Z. (2018) Concurrent and Temporal Relationships Between Humility and Emotional and Psychological Well-Being. Journal of Happiness Studies, 1-16. doi:10.1007/s10902s

Tough, H., Siegrist, J., y Fekete, C. (2017). Social relationships, mental health and wellbeing in physical disability: a systematic review. BMC Public Health, 17(1), 1-18.doi:10.1186/s12889-0174308-6.

Van Dierendonck, D. (2004) The construct validity of Ryff's Scale of Psychological well-being and its extension with spiritual well-being. Personality and Individual Differences, 36(3), 629-644. doi:10.1016/S0191-8869(03)00122-3

Velásquez, C., Montgomery, W., Montero, V., Pomalaya, R., Dioses, A., Velásquez, N., Araki, R. y Reynoso, D. (2008). Bienestar psicológico, asertividad y rendimiento académico en estudiantes universitarios sanmarquinos. Revista de Investigación en Psicología, 11(2), 139-152. doi:10.15381/rinvp.v11i2.3845 
Alós Cívico et al.

Zubieta, E. y Delfino, G. (2010). Satisfacción con la vida, bienestar psicológico y bienestar social en estudiantes universitarios de Buenos Aires. Anuario de Investigaciones, 17, 277-283.

Recibido: 26-05-2020

Acceptado: $16-11-2020$ 